\title{
A P7 Phage-Like Plasmid Carrying mcr-1 in an ST15 Klebsiella pneumoniae Clinical Isolate
}

\author{
Weilong Zhou ${ }^{1,2+}$, Lu Liu ${ }^{1,2+}$, Yu Feng ${ }^{1,2}$ and Zhiyong Zong 1,2,3,4* \\ ${ }^{1}$ Center of Infectious Diseases, West China Hospital, Sichuan University, Chengdu, China, ${ }^{2}$ Division of Infectious Diseases, \\ State Key Laboratory of Biotherapy, Chengdu, China, ${ }^{3}$ Department of Infection Control, West China Hospital, Sichuan \\ University, Chengdu, China, ${ }^{4}$ Center for Pathogen Research, West China Hospital, Sichuan University, Chengdu, China
}

\section{OPEN ACCESS}

Edited by:

Raffaele Zarrilli,

Department of Public Health,

University of Naples Federico II, Italy

Reviewed by:

Remy A. Bonnin,

Université Paris-Saclay, France

Jason Sahl,

Northern Arizona University,

United States

Antonio Cannatelli,

University of Siena, Italy

Davide Sassera,

University of Pavia, Italy

${ }^{*}$ Correspondence:

Zhiyong Zong

zongzhiy@scu.edu.cn

${ }^{\dagger}$ These authors have contributed equally to this work.

Specialty section

This article was submitted to Antimicrobials, Resistance and

Chemotherapy

a section of the journal

Frontiers in Microbiology

Received: 24 September 2017 Accepted: 05 January 2018

Published: 22 January 2018

Citation:

Zhou W, Liu L, Feng Y and Zong Z (2018) A P7 Phage-Like Plasmid Carrying mcr-1 in an ST15 Klebsiella

pneumoniae Clinical Isolate.

Front. Microbiol. 9:11

doi: 10.3389/fmicb.2018.00011
A Klebsiella pneumoniae clinical strain, named SCKP83, was isolated and found to be resistant to colistin thanks to the presence plasmid-borne colistin resistant gene $\mathrm{mcr}-1$. The strain was subjected to whole genome sequencing and conjugation experiments. The subsequent analysis indicated that the strain belongs to ST15 and the capsular type K41. In SCKP83, mcr-1 was carried by a 97.4-kb non-self-transmissible plasmid, a 90.9-kb region of which was predicted as an intact phage. This phage was $47.79 \%$ GC content, encoded 105 proteins and contained three tRNAs. mcr-1 was located downstream of two copies of the insertion sequence ISAp/1 (one complete and one truncated) and was inserted in the ant1 gene, which encodes a putative antirepressor for antagonizing $\mathrm{C} 1$ repression, in this phage. The phage is highly similar to phage P7 (77\% coverage and 98\% identity) from Escherichia coli. Several similar mcr-1-carrying plasmids have been found in E. coli at various locations in China, suggesting that these phage-like plasmids have circulated in China. The findings in this study suggest that the $\mathrm{P} 7$ phage-like plasmids are not restricted to $E$. coli and may represent new vehicles to mediate the inter-species spread of $m c r-1$.

Keywords: colistin, resistance, phagemid, plasmids, Klebsiella pneumoniae

\section{INTRODUCTION}

Klebsiella pneumoniae is a major pathogen causing a variety of infections in humans. Colistin is the last resort antimicrobial agent to treat infections caused by $K$. pneumoniae including those with resistance to carbapenems. However, colistin-resistant $K$. pneumoniae have emerged worldwide (Olaitan et al., 2014a). A few mechanisms including both chromosomal and plasmid-borne ones have been identified to be responsible for resistance to colistin in K. pneumoniae (Olaitan et al., 2014b). Plasmid-borne colistin resistance genes including $m c r-1$ (Liu et al., 2016), $m c r-2$ (Xavier et al., 2016), and $m c r-3$ (Yin et al., 2017) have been found recently. In particular, $m c r-1$ has been identified in various species of the Enterobacteriaceae in many countries (Poirel et al., 2017).

Bacteriophages (phages) are viruses able to infect and replicate within bacteria. Phages mediate the transfer of genetic components between bacteria via transduction. Phages may have a lytic cycle or a lysogenic cycle or both. In the lytic cycle, phage genomes are replicated and are assembled into particles, which cause cell lysis and are then released. In the lysogenic cycle, phage genomes integrate into the chromosome of host bacterial cells to exist in a latent or dormant state without causing cell lysis (Feiner et al., 2015). The structure of phages typically consists of a protein head 
that encapsulates a DNA or RNA genome and a tail that attacks the bacterial host (Wurtz, 1992). Phage genomes vary remarkbly in form and size but usually encode products for host takeover, replication, virion assembly, or lysis (Black and Thomas, 2012). Some phages may integrate into plasmids and can therefore be transferred by the host plasmid (Oliver et al., 2005; Shin and Ko, 2015).

$m c r-1$ is commonly carried by plasmids of the IncI 2 or IncX 4 replicon type and has also been found on IncF, IncHI2, or IncP plasmids (Poirel et al., 2017). We have found a plasmid carrying mcr-1 and phage P7-like sequences, which is reported here.

\section{METHODS}

\section{Bacterial Strain}

K. pneumoniae strain SCKP83 was recovered from a sputum sample of a 90-year-old male patient with severe pneumonia in February 2017 in China, who did not receive colistin before. Species identification was performed using Vitek II (bioMérieux, Marcy-l'Étoile, France) and MALTI-TOF (Bruker, Billerica, MA, USA). In vitro susceptibility of colistin was performed using the broth dilution method of the Clinical Laboratory Standards Institute (CLSI) (CLSI, 2017) and breakpoints of colistin defined by EUCAST (http://www.eucast.org/) were applied. The presence of plasmid-borne colistin resistant genes $m c r-1, m c r-2$, and $m c r-3$ was screened by PCR as described previously (Xavier et al., 2016; Zhao and Zong, 2016; Yin et al., 2017).

\section{Whole Genome Sequencing and Analysis}

The strain was subjected to whole genome sequencing. Genomic DNA was prepared using the QIAamp DNA Mini Kit (Qiagen, Hilden, Germany) and whole genome sequencing was performed using the HiSeq X10 Sequencer (Illumina, San Diego, CA). The coverage was approximately $300 \times$ coverage, which was calculated based on the estimated genome size and the average output of the sequencer. Reads were trimmed using Trimmomatic (version 0.36) (Bolger et al., 2014) and were then assembled to contigs using SPAdes (version 3.11) (Bankevich et al., 2012) with careful mode turned on. Sequence type and capsular type were determined using the genomic sequence to query the multi-locus sequence typing and $w z i$ allele databases of $K$. pneumoniae available at http://bigsdb.pasteur. fr/klebsiella/klebsiella.html. Antimicrobial resistance genes were identified from genome sequences using the ABRicate program (https://github.com/tseemann/abricate) and ResFinder (https:// cge.cbs.dtu.dk/services/ResFinder/). The plasmid carrying mcr1, designated pMCR_SCKP-LL83, was circularized using PCR and Sanger sequencing to fill in gaps between contigs. Plasmid replicon was determined using the PlasmidFinder tool at http:// genomicepidemiology.org/. Similar plasmids were retrieved from the GenBank and pairwise comparisons were preformed using BLASTn alignment (Altschul et al., 1990) and BRIG (Alikhan et al., 2011). The presence of phages was screened using PHASTER (http://phaster.ca/) (Arndt et al., 2016). tRNAs were screened using tRNA-SE (http://lowelab.ucsc.edu/tRNAscanSE/) (Lowe and Chan, 2016).

\section{Nucleotide Sequence Accession Numbers}

Draft whole-genome sequence of strain SCKP83 has been deposited into GenBank under the accession number NOKM00000000. Short reads of the whole-genome sequence of strain SCKP83 has been deposited into Short Reads Achieve under the accession number SRP099296. The complete sequences of pMCR_SCKP-LL83 has been deposited into GenBank under the accession number MF510496.

\section{Conjugation and Transformation Experiments}

Conjugation experiments were performed using both broth- and filter-based methods as described previously (Coque et al., 2002; Novais et al., 2006; Valenzuela et al., 2007). The azide-resistant Escherichia coli strain J53 was used as the recipient and $2 \mu \mathrm{g} / \mathrm{ml}$ colistin plus $150 \mu \mathrm{g} / \mathrm{ml}$ sodium azide were used for selecting transconjugants. Plasmids were prepared from strain SCKP83 using alkaline lysis (Sambrook and Russell, 2001) and were used for electroporation. Electroporation was conducted using a Gene Pulser (Bio-Rad, Hercules, CA, USA) with an electrical pulse of $25 \mu \mathrm{F}$ capacitance, $2.5 \mathrm{kV}$ and $200 \Omega$ sample resistance. E. coli strain $\mathrm{DH} 5 \alpha$ and a colistin-susceptible K. pneumoniae strain 020018 were used as recipient strains. Potential transformants were selected on agar plates containing $2 \mu \mathrm{g} / \mathrm{ml}$ colistin.

\section{Induction of Bacteriophage}

To determine the nature of pMCR_SCKP-LL83, we performed the induction assay using ultraviolet ray and mitomycin $\mathrm{C}$ as described previously (Mitsui et al., 1973; Raya and H’bert, 2009). Briefly, for UV induction, $1 \mathrm{ml}$ culture of strain SCKP-LL83 in the exponential phase was harvested and resuspended in $0.05 \mathrm{M}$ phosphate buffer ( $\mathrm{pH}$ 6.8). The suspension was adjusted to the

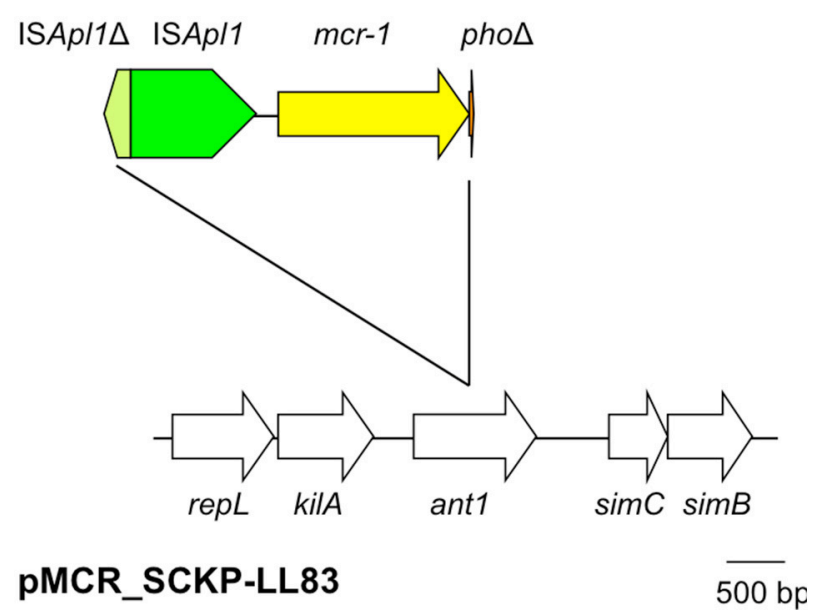

FIGURE 1 | The genetic context of mor-1 on pMCR_SCKP-LL83. The ISAp/1 $\Delta$-ISAp/1-mcr-1-pho $\Delta$ structure is inserted into ant1 but without the 2-bp DR characteristic of the insertion of ISAp/1. The two ISAp/1 are at contrary oppositions. $\Delta$ refers to truncated genes or elements. ant1 encodes a putative antirepressor. The phage genes surrounding ant1 include repL (encoding replication protein), kliA (encoding a putative host killing protein), $\operatorname{sim} B$ and $\operatorname{sim} C$ (both encoding proteins for host immunity). 
TABLE 1 | Features of pMCR_SCKP-LL83.

\begin{tabular}{|c|c|c|}
\hline Feature $^{a}$ & $\begin{array}{l}\text { Position } \\
\text { (start-end) }\end{array}$ & Function \\
\hline 0001 & $356-1912$ & $\begin{array}{l}\text { Type I restriction-modification system } \\
\text { subunit M }\end{array}$ \\
\hline 0002 & 1909-3114 & Restriction endonuclease subunit S \\
\hline 0003 & $3235-6351$ & $\begin{array}{l}\text { Type I restriction enzyme EcoR124|| R } \\
\text { protein }\end{array}$ \\
\hline 0004 & $6616-7122$ & $3^{\prime}$-Phosphatase, $5^{\prime}$-polynucleotide kinase \\
\hline 0005/pmgs & $7195-8457$ & Putative morphogenetic protein \\
\hline 0006 & $8459-8677$ & Hypothetical protein \\
\hline 0007 & $8759-9460$ & Hypothetical protein \\
\hline 0008/pphA & $9457-10134$ & Serine/Threonine protein phosphatase \\
\hline 0009/pmgP & $10131-10757$ & Putative morphogenetic protein \\
\hline 0010 & $11259-11414$ & Hypothetical protein \\
\hline 0011/pmgM & $11481-12059$ & Putative morphogenetic function protein \\
\hline 0012 & $12062-12307$ & Putative morphogenetic protein \\
\hline 0013 & $12571-12831$ & Baseplate protein \\
\hline 0014 & $12841-14058$ & Tail protein \\
\hline 0015 & $14062-14790$ & Tail protein \\
\hline 0016 & $14777-15562$ & Hypothetical protein \\
\hline 0017 & $15564-16580$ & Tail length tape measure protein \\
\hline 0018 & 16573-17205 & Putative baseplate protein \\
\hline 0019 & $17252-18250$ & Hypothetical protein \\
\hline 0018/dnaB & 18250-19614 & Replicative DNA helicase \\
\hline 0021 & 19900-19975 & tRNA-Met \\
\hline 0024/tciA & 20250-20675 & $\begin{array}{l}\text { Putative tellurite or colicin resistance } \\
\text { protein }\end{array}$ \\
\hline 0025 & $21187-21360$ & Hypothetical protein \\
\hline 0026 & 21603-21678 & tRNA-Thr \\
\hline 0027 & $21681-21756$ & tRNA-Asn \\
\hline $0028 / d m t$ & $22429-24693$ & DNA adenine methylase family protein \\
\hline $0029 / r d g C$ & $24690-25595$ & Recombination-associated protein RdgC \\
\hline 0030 & 25588-25872 & Hypothetical protein \\
\hline 0031 & $25857-26096$ & Hypothetical protein \\
\hline 0032 & 26335-27123 & Hypothetical protein \\
\hline 0033 & 27163-27585 & Outer membrane lytic protein \\
\hline 0034/upfB & 27763-28155 & Hypothetical protein \\
\hline 0035 & 28048-28311 & Hypothetical protein \\
\hline 0036/repA & $28491-29375$ & $\begin{array}{l}\text { Initiator replication family protein of } \\
\text { p0111-like replicon }\end{array}$ \\
\hline 0037 & 29668-30477 & Helicase \\
\hline IS1294 & 32106-32205 & Insertion sequence \\
\hline 0040/parA & $32334-33530$ & Plasmid partition protein $\mathrm{A}$ \\
\hline 0041/parB & $33547-34548$ & Plasmid partition protein B \\
\hline 0042 & $34774-36480$ & Putative baseplate protein \\
\hline 0043 & $36541-38130$ & Hypothetical protein \\
\hline 0044 & 38140-38955 & Tail tube protein \\
\hline 0045/pmgG & 38991-39572 & Putative morphogenetic protein \\
\hline 0046/bp/B & $39584-40093$ & Putative baseplate structural protein \\
\hline 0047 & $40217-40423$ & Hypothetical protein \\
\hline 0048 & $40547-40792$ & Hypothetical protein \\
\hline 0049/repL & $40843-41652$ & Replication protein \\
\hline 0050/kilA & $41718-42518$ & Putative host killing protein \\
\hline
\end{tabular}

TABLE 1 | Continued

\begin{tabular}{|c|c|c|}
\hline Feature ${ }^{a}$ & $\begin{array}{l}\text { Position } \\
\text { (start-end) }\end{array}$ & Function \\
\hline 0051 & $42682-43587$ & Hypothetical protein \\
\hline 0052/mcr-1 & $43541-45166$ & Colistin resistance \\
\hline ISAp/1 & $45353-46422$ & Insertion sequence \\
\hline ISAp/1 $\Delta$ & $46423-46645$ & Insertion sequence, truncated \\
\hline 0055 & $46580-46915$ & Antirepressor protein \\
\hline 0056 & $46912-47133$ & Hypothetical protein \\
\hline $0057 / \operatorname{sim} B$ & $47561-48031$ & Superimmunity linked function \\
\hline 0058/simC & $48039-48818$ & Superimmunity linked function \\
\hline 0059/pmgC & 49028-49594 & putative morphogenetic protein \\
\hline 0060/tubB & $49605-50216$ & Major tail tube protein \\
\hline 0061/pmgB & $50231-51112$ & Putative morphogenetic protein \\
\hline 0062 & $51194-54586$ & Transglycosylase SLT domain protein \\
\hline 0063/pmgA & $54586-54942$ & Putative morphogenetic protein \\
\hline 0064 & 54939-56372 & Putative baseplate structural protein \\
\hline 0065 & $56372-57208$ & Putative tail tube protein \\
\hline 0066 & $57287-57721$ & $\begin{array}{l}\text { Putative tail fiber structure or assembly } \\
\text { protein }\end{array}$ \\
\hline 0067 & $57733-59214$ & Hypothetical protein \\
\hline 0068 & $59483-59728$ & Hypothetical protein \\
\hline 0069 & 59769-60206 & Hypothetical protein \\
\hline 0070 & $60217-60645$ & Hypothetical protein \\
\hline 0071 & $60686-61159$ & Hypothetical protein \\
\hline 0072 & 61188-61646 & Hypothetical protein \\
\hline 0073/tfaE & $62160-62771$ & Prophage tail fiber assembly protein $\mathrm{TfaE}$ \\
\hline 0074 & $62771-63229$ & Hypothetical protein \\
\hline 0075 & $63240-63683$ & Hypothetical protein \\
\hline 0076/pin & $63773-64345$ & Site-specific recombinase \\
\hline 0077 & $64781-65044$ & Hypothetical protein \\
\hline 0078/lydA & $65119-65448$ & Lysis determining protein \\
\hline 0079 & $65445-65888$ & Lysis determining protein \\
\hline 0080 & $65875-66477$ & Hypothetical protein \\
\hline 0081/darA & 66479-68398 & Hypothetical protein \\
\hline $0082 / d d r A$ & $68395-68760$ & Hypothetical protein \\
\hline 0083 & $68797-71760$ & Hypothetical protein \\
\hline $0084 / h \times r$ & 71750-72061 & Putative repressor protein $\mathrm{Hxr}$ \\
\hline 0085/ompD & $72804-73916$ & Outer membrane porin protein OmpD \\
\hline 0086/ssb & $74150-74638$ & Single-stranded DNA-binding protein \\
\hline 0087/lys & $74808-75365$ & Lysozyme \\
\hline 0088 & $75657-76676$ & Putative head processing protein \\
\hline 0089 & 76669-78378 & Putative portal protein \\
\hline 0090 & $78454-85221$ & Putative DNA adenine methyltransferase \\
\hline 0091 & $85255-85695$ & Hypothetical protein \\
\hline 0092 & $85692-85940$ & Modulator protein \\
\hline 0093 & $85982-87286$ & Hypothetical protein \\
\hline 0094 & $87343-87984$ & Maturation control protein \\
\hline 0095/ref & $88173-88733$ & $\begin{array}{l}\text { Recombination enhancement function } \\
\text { protein }\end{array}$ \\
\hline 0096 & $88981-89190$ & Putative lysogeny establishment protein \\
\hline 0097/cre & 89343-90374 & GST-loxP-cre recombinase fusion protein \\
\hline 0098/cra & 90382-90603 & Putative Cre-associated regulatory protein \\
\hline
\end{tabular}


TABLE 1 | Continued

\begin{tabular}{|c|c|c|}
\hline Feature $^{a}$ & $\begin{array}{l}\text { Position } \\
\text { (start-end) }\end{array}$ & Function \\
\hline 0099 & $91208-91417$ & C1 repressor inactivator \\
\hline 0100 & $91528-92379$ & Primary repressor of lytic function \\
\hline 0101 & $92405-93889$ & Putative large terminase protein \\
\hline 102/pacA & 93889-95082 & Terminase A protein \\
\hline 0103/lpa & $95169-95621$ & Late promoter activating protein \\
\hline 0104 & $95710-96753$ & Hypothetical protein \\
\hline 0105 & $96781-96960$ & Hypothetical protein \\
\hline 0106/doc & $96965-97345$ & Toxin Doc \\
\hline 0001 & $356-1912$ & $\begin{array}{l}\text { Type I restriction-modification system } \\
\text { subunit M }\end{array}$ \\
\hline 0002 & 1909-3114 & Restriction endonuclease subunit S \\
\hline 0003 & $3235-6351$ & $\begin{array}{l}\text { Type I restriction enzyme EcoR124II R } \\
\text { protein }\end{array}$ \\
\hline 0004 & $6616-7122$ & 3'-Phosphatase, $5^{\prime}$-polynucleotide kinase \\
\hline 0005/pmgs & $7195-8457$ & Putative morphogenetic protein \\
\hline 0006 & 8459-8677 & Hypothetical protein \\
\hline 0007 & $8759-9460$ & Hypothetical protein \\
\hline 0008/pphA & $9457-10134$ & Serine/Threonine protein phosphatase \\
\hline 0009/pmgP & $10131-10757$ & Putative morphogenetic protein \\
\hline 0010 & $11259-11414$ & Hypothetical protein \\
\hline 0011/pmgM & $11481-12059$ & Putative morphogenetic function protein \\
\hline 0012 & $12062-12307$ & Putative morphogenetic protein \\
\hline 0013 & $12571-12831$ & Baseplate protein \\
\hline 0014 & $12841-14058$ & Tail protein \\
\hline 0015 & $14062-14790$ & Tail protein \\
\hline 0016 & $14777-15562$ & Hypothetical protein \\
\hline 0017 & $15564-16580$ & Tail length tape measure protein \\
\hline 0018 & $16573-17205$ & Putative baseplate protein \\
\hline 0019 & $17252-18250$ & Hypothetical protein \\
\hline 0018/dnaB & $18250-19614$ & Replicative DNA helicase \\
\hline 0021 & $19900-19975$ & tRNA-Met \\
\hline 0024/tciA & 20250-20675 & $\begin{array}{l}\text { Putative tellurite or colicin resistance } \\
\text { protein }\end{array}$ \\
\hline 0025 & $21187-21360$ & Hypothetical protein \\
\hline 0026 & 21603-21678 & tRNA-Thr \\
\hline 0027 & $21681-21756$ & tRNA-Asn \\
\hline $0028 / d m t$ & $22429-24693$ & DNA adenine methylase family protein \\
\hline 0029/rdgC & $24690-25595$ & Recombination-associated protein RdgC \\
\hline 0030 & $25588-25872$ & Hypothetical protein \\
\hline 0031 & $25857-26096$ & Hypothetical protein \\
\hline 0032 & 26335-27123 & Hypothetical protein \\
\hline 0033 & $27163-27585$ & Outer membrane lytic protein \\
\hline 0034/upfB & 27763-28155 & Hypothetical protein \\
\hline 0035 & $28048-28311$ & Hypothetical protein \\
\hline 0036/repA & 28491-29375 & $\begin{array}{l}\text { Initiator replication family protein of } \\
\text { p0111-like replicon }\end{array}$ \\
\hline 0037 & 29668-30477 & Helicase \\
\hline IS1294 & 32106-32205 & Insertion sequence \\
\hline 0040/parA & $32334-33530$ & Plasmid partition protein A \\
\hline 0041/parB & $33547-34548$ & Plasmid partition protein B \\
\hline 0042 & $34774-36480$ & Putative baseplate protein \\
\hline
\end{tabular}

TABLE 1 | Continued

\begin{tabular}{|c|c|c|}
\hline Feature $^{a}$ & $\begin{array}{l}\text { Position } \\
\text { (start-end) }\end{array}$ & Function \\
\hline 0043 & $36541-38130$ & Hypothetical protein \\
\hline 0044 & 38140-38955 & Tail tube protein \\
\hline 0045/pmgG & 38991-39572 & Putative morphogenetic protein \\
\hline 0046/bplB & $39584-40093$ & Putative baseplate structural protein \\
\hline 0047 & $40217-40423$ & Hypothetical protein \\
\hline 0048 & $40547-40792$ & Hypothetical protein \\
\hline 0049/repL & $40843-41652$ & Replication protein \\
\hline 0050/kilA & $41718-42518$ & Putative host killing protein \\
\hline 0051 & $42682-43587$ & Hypothetical protein \\
\hline 0052/mcr-1 & $43541-45166$ & Colistin resistance \\
\hline ISAp/1 & $45353-46422$ & Insertion sequence \\
\hline ISAp/1 $\Delta$ & $46423-46645$ & Insertion sequence, truncate \\
\hline 0055 & $46580-46915$ & Antirepressor protein \\
\hline 0056 & $46912-47133$ & Hypothetical protein \\
\hline $0057 / \operatorname{sim} B$ & $47561-48031$ & Superimmunity linked function \\
\hline 0058/sim C & $48039-48818$ & Superimmunity linked function \\
\hline 0059/pmgC & 49028-49594 & Putative morphogenetic protein \\
\hline 0060/tubB & $49605-50216$ & Major tail tube protein \\
\hline 0061/pmgB & $50231-51112$ & Putative morphogenetic protein \\
\hline 0062 & $51194-54586$ & Transglycosylase SLT domain protein \\
\hline 0063/pmgA & $54586-54942$ & putative morphogenetic protein \\
\hline 0064 & 54939-56372 & putative baseplate structural protein \\
\hline 0065 & $56372-57208$ & Putative tail tube protein \\
\hline 0066 & $57287-57721$ & $\begin{array}{l}\text { Putative tail fiber structure or assembly } \\
\text { protein }\end{array}$ \\
\hline 0067 & $57733-59214$ & Hypothetical protein \\
\hline 0068 & $59483-59728$ & Hypothetical protein \\
\hline 0069 & 59769-60206 & Hypothetical protein \\
\hline 0070 & $60217-60645$ & Hypothetical protein \\
\hline 0071 & $60686-61159$ & Hypothetical protein \\
\hline 0072 & $61188-61646$ & Hypothetical protein \\
\hline 0073/tfaE & $62160-62771$ & Prophage tail fiber assembly protein $\mathrm{TfaE}$ \\
\hline 0074 & $62771-63229$ & Hypothetical protein \\
\hline 0075 & $63240-63683$ & Hypothetical protein \\
\hline 0076/pin & $63773-64345$ & Site-specific recombinase \\
\hline 0077 & $64781-65044$ & Hypothetical protein \\
\hline $0078 / / y d A$ & $65119-65448$ & Lysis determining protein \\
\hline 0079 & $65445-65888$ & Lysis determining protein \\
\hline 0080 & $65875-66477$ & Hypothetical protein \\
\hline 0081/darA & 66479-68398 & Hypothetical protein \\
\hline 0082/ddrA & $68395-68760$ & Hypothetical protein \\
\hline 0083 & $68797-71760$ & Hypothetical protein \\
\hline $0084 / h \times r$ & $71750-72061$ & Putative repressor protein $\mathrm{Hxr}$ \\
\hline 0085/ompD & $72804-73916$ & Outer membrane porin protein OmpD \\
\hline $0086 / s s b$ & $74150-74638$ & Single-stranded DNA-binding protein \\
\hline 0087/lys & $74808-75365$ & Lysozyme \\
\hline 0088 & $75657-76676$ & Putative head processing protein \\
\hline 0089 & 76669-78378 & Putative portal protein \\
\hline 0090 & $78454-85221$ & Putative DNA adenine methyltransferase \\
\hline 0091 & $85255-85695$ & Hypothetical protein \\
\hline 0092 & 85692-85940 & Modulator protein \\
\hline
\end{tabular}


TABLE 1 | Continued

\begin{tabular}{lll}
\hline Feature & \multicolumn{1}{c}{$\begin{array}{c}\text { Position } \\
\text { (start-end) }\end{array}$} & Function \\
\hline 0093 & $85982-87286$ & Hypothetical protein \\
0094 & $87343-87984$ & Maturation control protein \\
0095/ref & $88173-88733$ & Recombination enhancement function \\
& & protein \\
0096 & $88981-89190$ & Putative lysogeny establishment protein \\
0097/cre & $89343-90374$ & GST-loxP-cre recombinase fusion protein \\
0098/cra & $90382-90603$ & Putative Cre-associated regulatory protein \\
0099 & $91208-91417$ & C1 repressor inactivator \\
0100 & $91528-92379$ & Primary repressor of lytic function \\
0101 & $92405-93889$ & Putative large terminase protein \\
102/pacA & $93889-95082$ & Terminase A protein \\
0103//pa & $95169-95621$ & Late promoter activating protein \\
0104 & $95710-96753$ & Hypothetical protein \\
0105 & $96781-96960$ & Hypothetical protein \\
$0106 /$ doc & $96965-97345$ & Toxin Doc \\
\hline
\end{tabular}

a Features: genes, mobile genetic elements or $C$-segments. The allele numbers of genes present on PMCR_SCKP-LL83 are shown.

$0.5 \mathrm{McFarland}$ turbidity. Six aliquots of $150 \mu \mathrm{l}$ were spotted on a $9 \mathrm{~cm}$ Petri dish and irradiated by a germicidal UV lamp at a distance of $100 \mathrm{~cm}$. The drops were collected at 10, 20, 30, 60, 90, and $120 \mathrm{~s}$ serially, each of which was then incubated with $1 \mathrm{ml} \mathrm{LB}$ broth under $37^{\circ} \mathrm{C}$ in dark for 3-4 h. Lysis was observed by naked eyes. For mitomycin $\mathrm{C}$ induction, $100 \mathrm{ml}$ cultures of strain SCKPLL83 were added with mitomycin $\mathrm{C}$ to a final concentration of $0.1,1,10,20$, and $40 \mu \mathrm{g} / \mathrm{ml}$ and were incubated under $37^{\circ} \mathrm{C}$ with shaking. Aliquots $(1 \mathrm{ml})$ were sampled at $2,4,12$, and $24 \mathrm{~h}$. The cultures were filtrated through $0.22 \mu \mathrm{m}$ polyethersulfone membranes (Merck Millipore, Billerica, MA, USA) and the membranes were used for the plaque formation test, which was carried out via the agar overlay method (Kropinski et al., 2009). All of the tests were performed in triplicate.

\section{Assay for Replication Module}

The replication initiation protein-encoding gene repB and its replication origin sequence (ori) of pMCR_SCKP-LL83 were amplified with self-designed primers OriF (CGGAATTCGAAAT GGGATCAACATTGACTATACG) and OriR (CGGAATTCAT CAATACCACTGCTTGATGAGA; EcoRI sites are underlined). The amplicons were cloned onto the vector pKC1139, which has a temperature sensitive origin oriT and cannot replicate at temperatures higher than $30^{\circ} \mathrm{C}$. The ligated vectors were transformed into $E$. coli $\mathrm{DH} 5 \alpha$ and the transformants were screened by apramycin $(100 \mu \mathrm{g} / \mathrm{ml})$ at $37^{\circ} \mathrm{C}$. The presence of rep $B$ and ori in transformants were confirmed by PCR with M13 (-21) Forward and M13-R primers binding to the clone vector and Sanger sequencing.

\section{RESULTS AND DISCUSSION}

Strain SCKP83 was resistant to colistin (MIC, $8 \mu \mathrm{g} / \mathrm{ml}$ ) and had $m c r-1$ but no $m c r-2$ and $m c r-3$ genes. Whole genome sequencing of strain SCKP83 generated 5,247,124 clean reads, which were then assembled to 119 contigs $(89>1,000 \mathrm{bp})$ with a $50.38 \%$ GC content. Strain SCKP83 belonged to ST15, which is a relative common type of $K$. pneumoniae seen in China (Zhang et al., 2017b). The capsular type of strain SCKP83 was K41.

mor-1 was carried by a $97.4 \mathrm{~kb}$ plasmid, pMCR_SCKPLL83, which did not carry any additional known antimicrobial resistance genes. Despite repeated attempts, no colistin resistant transconjugants were obtained, suggesting that pMCR_SCKPLL83 is not self-transmissible. In addition, the transformation of this plasmid into E. coli strain $\mathrm{DH} 5 \alpha$ and a colistin-susceptible $K$. pneumoniae strain was unsuccessful. This suggests that this plasmid may be strain-specific or its transformation occurs at a low frequency, which could not be detected in our experiments. pMCR_SCKP-LL83 had a single pO111 plasmid replicon. Transformants containing repB and its ori were obtained. The presence of $r e p B$ and ori allows the temperature sensitive vector pKC1139 to replicate at $37^{\circ} \mathrm{C}$, suggesting that the replication module of pMCR_SCKP-LL83 indeed leads to the replication of this plasmid.

On pMCR_SCKP-LL83, $m c r-1$ was located downstream of a complete insertion sequence ISApl1. The phosphoesteraseencoding pho gene that is always located downstream of mcr-1 was truncated at its 3 '-end with only 38 bp out of the 747-bp gene remaining. Surprisingly, immediate upstream of the complete ISApl1 (1,070 bp in length) lies another ISApl1 that is truncated at its $5^{\prime}$-end with the presence of $223 \mathrm{bp}$ including an intact right-hand inverted repeat (IRR) (Figure 1). When we artificially subtract the ISApl1 $\Delta$-ISApl1mcr-1-pho $\Delta$ region from pMCR_SCKP-LL83, the remaining artificially-joining sequence perfectly matched the ant1 gene, which encodes a putative antirepressor for antagonizing C1 repression by formation of Ant $1 / \mathrm{Ant} 2 / \mathrm{C} 1$ complex. It therefore becomes evident that the ISApl1 $\Delta$-ISApl1-mor-1-pho $\Delta$ structure is inserted into ant1. It has been found that a single copy of ISApl1 is able to mobilize $m c r-1$ and pho together with itself (Li et al., 2017; Zhao et al., 2017). The insertion of ISApl1 would generate 2-bp direct target repeats (DR). However, no 2-bp DRs were present flanking the ISApl1 $\Delta$-ISApl1-mcr-1-pho $\Delta$ structure, suggesting that the formation of such a complex structure was not directly due to the insertion mediated by ISApl1. The mechanism responsible for generating the ISApl1 $\Delta$ ISApl1-mor-1-pho $\Delta$ structure remains unclear but might have involved recombination.

A 90.9-kb region of the 97.4-kb pMCR_SCKP-LL83 was predicted as an intact phage. Neither the appearance of lysis nor the formation of plaques was observed in the UV induction. In mitomycin $\mathrm{C}$ induction, no plaques were formed at the tested concentrations and intervals. These results suggest that pMCR_SCKP-LL83 was indeed a plasmid. Nonetheless, the phage region on pMCR_SCKP-LL83 had 47.79\% GC content, encoded 105 proteins and contained three tRNAs, i.e., tRNAAsn, tRNA-Thr, and tRNA-Met (Table 1). pMCR_SCKP-LL83 is highly similar (72\% coverage and $98 \%$ identity) to the 101.7 kb Enterobacteria phage P7 (GenBank accession no. AF503408). Phage P7 (previously called as $\varphi a m p$ ) was isolated from E. coli of human fecal flora (Smith, 1972) and exists as a 


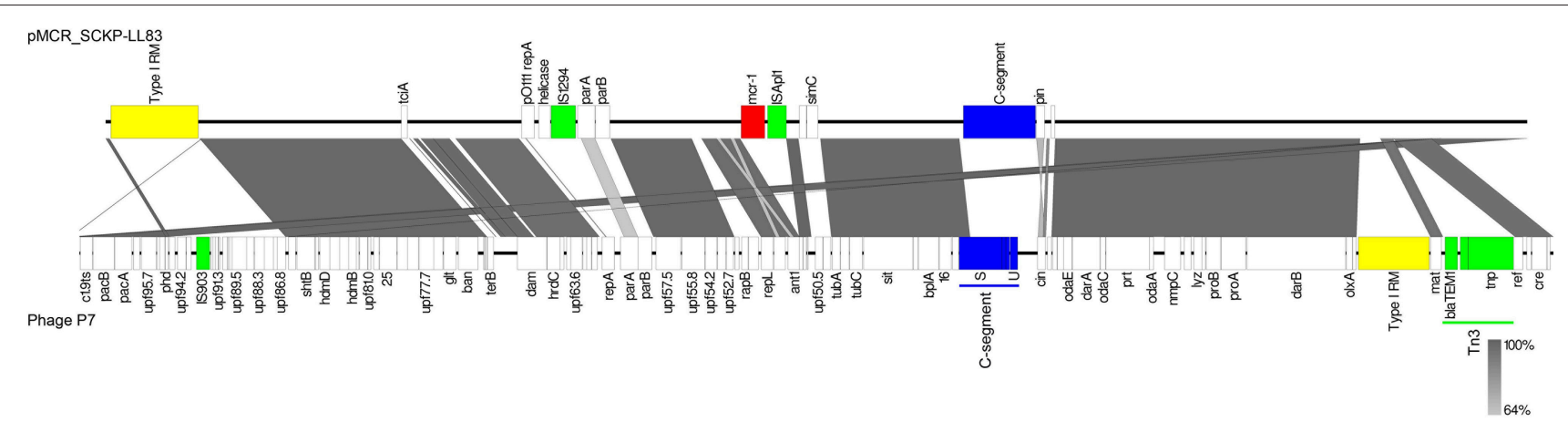

FIGURE 2 | Comparison of PMCR_SCKP-LL83 with phage P7 (GenBank accession no. AF503408). Similar regions are indicated with the degree of nucleotide identity being shown in gray scales. Mobile genetic elements, type I restriction-modification (RM) systems and C-segments are shown in green, yellow, and blue, respectively.

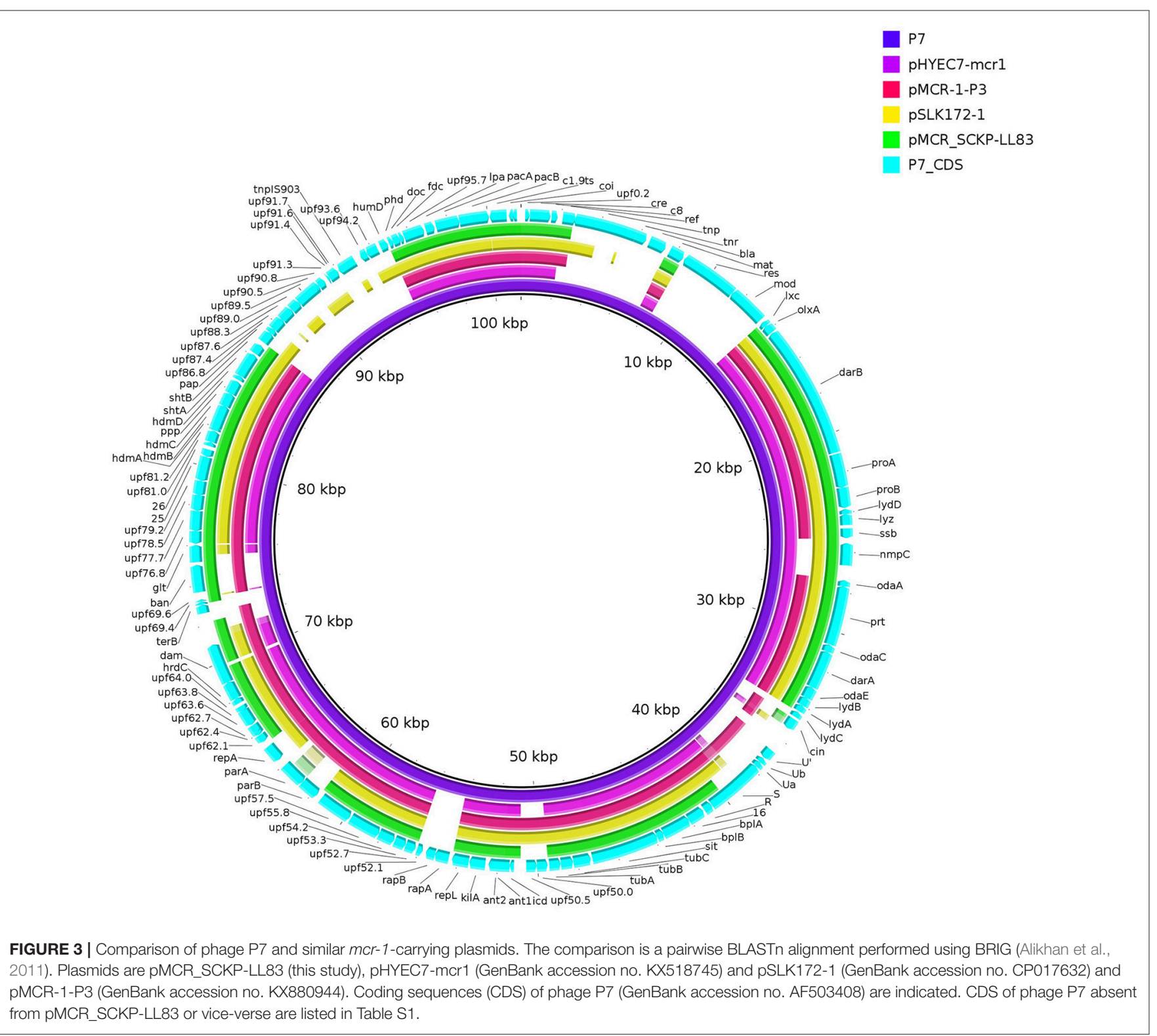


nonintegrated autonomous circular plasmid that constitutes a unique compatibility group (Hedges et al., 1975). Compared with P7, pMCR_SCKP-LL83 did not have the bla $a_{\text {TEM-1-carrying }}$ transposon Tn3, the type I restriction-modification system EcoP7, a 4-kb invertible C-segment and a few genes, most of which encode proteins of unknown function (Table S1 in the Supplementary file and Figure 2). C-segment contains several genes encoding phage tail fibers and also determines the host specificity of the phage (Iida, 1984). In contrast, pMCR_SCKPLL83 had a few extra genes including an unnamed type I restriction-modification system, $m c r-1$ and a $5-\mathrm{kb}$ putative invertible C-segment (Table S1), which is highly similar (92\% coverage and $99 \%$ identity) to the multiple DNA inversion region min on plasmid p15B of E. coli 15T (Sandmeier et al., 1991).

It is well known that phages can transfer genetic components between bacterial isolates, but the role of phages in disseminating antimicrobial resistance genes is still a matter of debate (Colavecchio et al., 2017; Enault et al., 2017). Nonetheless, some studies have found that phages are able to transfer genes conferring resistance to aminoglycosides ( $\operatorname{aadA}, \operatorname{aphA1}, \operatorname{str} A$, strB), $\beta$-lactams (bla $a_{\mathrm{CMY}-2}, b l a_{\mathrm{CTX}-\mathrm{M}-9}, b l a_{\mathrm{OXA}-2}, b l a_{\mathrm{OXA}-20}$, bla $\left.a_{\mathrm{PSE}-1}, b l a_{\mathrm{TEM}}\right)$, chloramphenicol $(f l o R)$, or tetracycline $($ tet $(\mathrm{A}), \operatorname{tet}(\mathrm{B}), \operatorname{tet} G$, tet $O$, tet $W$ ) via transduction (Zhang and LeJeune, 2008; Colomer-Lluch et al., 2014; Bearson and Brunelle, 2015; Ross and Topp, 2015; Shousha et al., 2015; Anand et al., 2016). In addition, a recent study has identified that two E. coli phages could promote the transformation of plasmids carrying antimicrobial resistance genes (Keen et al., 2017).

During the process of this work, $m c r-1$ in either complete or interrupted version has been found on plasmids containing similar phage sequences including pHYEC7-mcr1 (GenBank accession no. KX518745), pSLK172-1 (GenBank accession no. CP017632) (Bai et al., 2017), and pMCR-1-P3 (GenBank accession no. KX880944) (Zhang et al., 2017a). All of these plasmids have been recovered from $E$. coli at various locations of China and are highly similar (75-79\% coverage, 97-99\% identity, identified by BLAST; Figure 3) to pMCR_SCKP-LL83. This suggests that the phage sequence-containing plasmids represent new vehicles, which may have circulated in China, to mediate the spread of $m c r-1$ in addition to plasmids of IncI2, X4, F, HI2, and $\mathrm{P}$ types. The identification of PMCR_SCKP-LL83 from a

\section{REFERENCES}

Alikhan, N. F., Petty, N. K., Ben Zakour, N. L., and Beatson, S. A. (2011). BLAST Ring Image Generator (BRIG): simple prokaryote genome comparisons. BMC Genomics 12:402. doi: 10.1186/1471-2164-12-402

Altschul, S. F., Gish, W., Miller, W., Myers, E. W., and Lipman, D. J. (1990). Basic local alignment search tool. J. Mol. Biol. 215, 403-410. doi: 10.1016/S0022-2836(05)80360-2

Anand, T., Bera, B. C., Vaid, R. K., Barua, S., Riyesh, T., Virmani, N., et al. (2016). Abundance of antibiotic resistance genes in environmental bacteriophages. J. Gen. Virol. 97, 3458-3466. doi: 10.1099/jgv.0.000639

Arndt, D., Grant, J. R., Marcu, A., Sajed, T., Pon, A., Liang, Y., et al. (2016). PHASTER: a better, faster version of the PHAST phage search tool. Nucleic Acids Res. 44, W16-W21. doi: 10.1093/nar/gkw387

Bai, L., Wang, J., Hurley, D., Yu, Z., Wang, L., Chen, Q., et al. (2017). A novel disrupted $m c r-1$ gene and a lysogenized phage P1-like sequence detected from a large conjugative plasmid, cultured from a human atypical enteropathogenic
K. pneumoniae is worrisome, suggesting that the P7 phage-like plasmids are not restricted to E. coli and may involve in the interspecies spread of $m c r-1$. The various locations of $m c r-1$ on these plasmids suggest that these plasmids may have acquired $m c r-1$ independently.

In the previous study on the ability of E. coli phages to promote the transformation of plasmids carrying antimicrobial resistance gene, phages, and plasmids are separate entities (Keen et al., 2017), which are different from the phage-like plasmid in the present study. As mentioned above, the conjugation and transformation of pMCR_SCKP-LL83 were unsuccessful. Among phage-like plasmids carrying $m c r-1$, pMCR-1-P3 was not self-transmissible and there are no data about whether it can be transferred by transformation (Zhang et al., 2017a), while pSLK172-1 was self-transmissible (Bai et al., 2017). This suggests that some phage-like plasmids may have lost the conjugative module and are therefore not self-transmissible. It is possible that these plasmids acquire genes encoding the conjugative module to become self-transmissible.

In conclusion, we identified and characterized a mcr-1carrying P7 phage-like plasmid from a K. pneumoniae clinical isolate. Such phage-like plasmids may represent new types of vehicles to mediate the spread of $m c r-1$.

\section{AUTHOR CONTRIBUTIONS}

ZZ: designed the experiments, analyzed the data, and wrote the MS. LL: performed the experiments and analyzed the data. WZ and YF: analyzed the data.

\section{ACKNOWLEDGMENTS}

This work was supported by grants from the National Natural Science Foundation of China (project no. 81222025, 81572030, and 81661130159$)$.

\section{SUPPLEMENTARY MATERIAL}

The Supplementary Material for this article can be found online at: https://www.frontiersin.org/articles/10.3389/fmicb. 2018.00011/full\#supplementary-material

Escherichia coli (aEPEC) recovered in China. J. Antimicrob. Chemother. 72, 1531-1533. doi: 10.1093/jac/dkw564

Bankevich, A., Nurk, S., Antipov, D., Gurevich, A. A., Dvorkin, M., Kulikov, A. S., et al. (2012). SPAdes: a new genome assembly algorithm and its applications to single-cell sequencing. J. Comput. Biol. 19, 455-477. doi: $10.1089 / \mathrm{cmb} .2012 .0021$

Bearson, B. L., and Brunelle, B. W. (2015). Fluoroquinolone induction of phagemediated gene transfer in multidrug-resistant Salmonella. Int. J. Antimicrob. Agents 46, 201-204. doi: 10.1016/j.ijantimicag.2015.04.008

Black, L. W., and Thomas, J. A. (2012). Condensed genome structure. Adv. Exp. Med. Biol. 726, 469-487. doi: 10.1007/978-1-4614-0980-9_21

Bolger, A. M., Lohse, M., and Usadel, B. (2014). Trimmomatic: a flexible trimmer for Illumina sequence data. Bioinformatics 30, 2114-2120. doi: 10.1093/bioinformatics/btu170

CLSI (2017). "Performance Standards for Antimicrobial Susceptibility Testing; Twenty-Third Informational Supplement. M100-S27." (Wayne, PA: Clinical and Laboratory Standards Institute). 
Colavecchio, A., Cadieux, B., Lo, A., and Goodridge, L. D. (2017). Bacteriophages contribute to the spread of antibiotic resistance genes among foodborne pathogens of the enterobacteriaceae family - a review. Front. Microbiol. 8:1108. doi: 10.3389/fmicb.2017.01108

Colomer-Lluch, M., Calero-Cáceres, W., Jebri, S., Hmaied, F., Muniesa, M., and Jofre, J. (2014). Antibiotic resistance genes in bacterial and bacteriophage fractions of Tunisian and Spanish wastewaters as markers to compare the antibiotic resistance patterns in each population. Environ. Int. 73, 167-175. doi: 10.1016/j.envint.2014.07.003

Coque, T. M., Oliver, A., Perez-Diaz, J. C., Baquero, F., and Canton, R. (2002). Genes encoding TEM-4, SHV-2, and CTX-M-10 extended-spectrum $\beta$-lactamases are carried by multiple Klebsiella pneumoniae clones in a single hospital (Madrid, 1989 to 2000). Antimicrob. Agents Chemother. 46, 500-510. doi: 10.1128/AAC.46.2.500-510.2002

Enault, F., Briet, A., Bouteille, L., Roux, S., Sullivan, M. B., and Petit, M. A. (2017). Phages rarely encode antibiotic resistance genes: a cautionary tale for virome analyses. ISME J. 11, 237-247. doi: 10.1038/ismej.2016.90

Feiner, R., Argov, T., Rabinovich, L., Sigal, N., Borovok, I., and Herskovits, A. A. (2015). A new perspective on lysogeny: prophages as active regulatory switches of bacteria. Nat. Rev. Microbiol. 13, 641-650. doi: 10.1038/nrmicro3527

Hedges, R. W., Jacob, A. E., Barth, P. T., and Grinter, N. J. (1975). Compatibility properties of P1 and PhiAMP prophages. Mol. Gen. Genet. 141, 263-267. doi: $10.1007 /$ BF00341804

Iida, S. (1984). Bacteriophage P1 carries two related sets of genes determining its host range in the invertible C segment of its genome. Virology 134, 421-434. doi: 10.1016/0042-6822(84)90309-X

Keen, E. C., Bliskovsky, V. V., Malagon, F., Baker, J. D., Prince, J. S., Klaus, J. S., et al. (2017). Novel "Superspreader" bacteriophages promote horizontal gene transfer by transformation. MBio 8:e02115-16. doi: 10.1128/mBio.02115-16

Kropinski, A. M., Mazzocco, A., Waddell, T. E., Lingohr, E., and Johnson, R. P. (2009). Enumeration of bacteriophages by double agar overlay plaque assay. Methods Mol. Biol. 501:69. doi: 10.1007/978-1-60327-164-6_7

Li, R., Xie, M., Zhang, J., Yang, Z., Liu, L., Liu, X., et al. (2017). Genetic characterization of $m c r$-1-bearing plasmids to depict molecular mechanisms underlying dissemination of the colistin resistance determinant. J. Antimicrob. Chemother. 72, 393-401. doi: 10.1093/jac/dkw411

Liu, Y.-Y., Wang, Y., Walsh, T. R., Yi, L.-X., Zhang, R., Spencer, J., et al. (2016). Emergence of plasmid-mediated colistin resistance mechanism MCR-1 in animals and human beings in China: a microbiological and molecular biological study. Lancet Infect. Dis. 16, 161-168. doi: 10.1016/S1473-3099(15)00424-7

Lowe, T. M., and Chan, P. P. (2016). tRNAscan-SE On-line: integrating search and context for analysis of transfer RNA genes. Nucleic Acids Res. 44, W54-W57. doi: 10.1093/nar/gkw413

Mitsui, N., Kiritani, K., and Nishida, S. (1973). A Lysin(s) in lysates of Clostridium botulinum A190 induced by ultraviolet ray or Mitomycin C. Jpn. J. Microbiol. 17, 353-360. doi: 10.1111/j.1348-0421.1973.tb00786.x

Novais, A., Canton, R., Valverde, A., Machado, E., Galan, J. C., Peixe, L., et al. (2006). Dissemination and persistence of bla $a_{\mathrm{CTX}-\mathrm{M}-9}$ are linked to class 1 integrons containing CR1 associated with defective transposon derivatives from Tn402 located in early antibiotic resistance plasmids of IncHI2, IncP1- $\alpha$, and IncFI groups. Antimicrob. Agents Chemother. 50, 2741-2750. doi: 10.1128/AAC.00274-06

Olaitan, A. O., Diene, S. M., Kempf, M., Berrazeg, M., Bakour, S., Gupta, S. K., et al. (2014a). Worldwide emergence of colistin resistance in Klebsiella pneumoniae from healthy humans and patients in Lao PDR, Thailand, Israel, Nigeria and France owing to inactivation of the PhoP/PhoQ regulator $m g r B$ : an epidemiological and molecular study. Int. J. Antimicrob. Agents 44, 500-507. doi: 10.1016/j.ijantimicag.2014.07.020

Olaitan, A. O., Morand, S., and Rolain, J. M. (2014b). Mechanisms of polymyxin resistance: acquired and intrinsic resistance in bacteria. Front. Microbiol. 5:643. doi: $10.3389 /$ fmicb. 2014.00643

Oliver, A., Coque, T. M., Alonso, D., Valverde, A., Baquero, F., and Cantón, R. (2005). CTX-M-10 linked to a phage-related element is widely disseminated among Enterobacteriaceae in a Spanish hospital. Antimicrob. Agents Chemother. 49, 1567-1571. doi: 10.1128/AAC.49.4.1567-1571.2005
Poirel, L., Jayol, A., and Nordmann, P. (2017). Polymyxins: antibacterial activity, susceptibility testing, and resistance mechanisms encoded by plasmids or chromosomes. Clin. Microbiol. Rev. 30, 557-596. doi: 10.1128/CMR.00064-16

Raya, R. R., and H'bert, E. M. (2009). Isolation of phage via Induction of Lysogens. Methods Mol. Biol. 501, 23-32. doi: 10.1007/978-1-60327$164-6 \_3$

Ross, J., and Topp, E. (2015). Abundance of antibiotic resistance genes in bacteriophage following soil fertilization with dairy manure or municipal biosolids, and evidence for potential transduction. Appl. Environ. Microbiol. 81, 7905-7913. doi: 10.1128/AEM.02363-15

Sambrook, J., and Russell, D. W. (2001). Molecular Cloning. A Laboratory Manual. Cold Spring Harbour, NY: Cold Spring Harbour Laboratory Press.

Sandmeier, H., Iida, S., Hübner, P., Hiestand-Nauer, R., and Arber, W. (1991). Gene organization in the multiple DNA inversion region min of plasmid p15B of E. coli 15T-: assemblage of a variable gene. Nucleic Acids Res. 19, 5831-5838. doi: $10.1093 / \mathrm{nar} / 19.21 .5831$

Shin, J., and Ko, K. S. (2015). A plasmid bearing the bla $a_{\mathrm{CTX}-\mathrm{M}-15}$ gene and phage P1-like sequences from a sequence type 11 Klebsiella pneumoniae isolate. Antimicrob. Agents Chemother. 59, 6608-6610. doi: 10.1128/AAC.00265-15

Shousha, A., Awaiwanont, N., Sofka, D., Smulders, F. J., Paulsen, P., Szostak, M. P., et al. (2015). Bacteriophages isolated from chicken meat and the horizontal transfer of antimicrobial resistance genes. Appl. Environ. Microbiol. 81, 4600-4606. doi: 10.1128/AEM.00872-15

Smith, H. W. (1972). Ampicillin resistance in Escherichia coli by phage infection. Nat. New Biol. 238, 205-206. doi: 10.1038/newbio238205a0

Valenzuela, J. K., Thomas, L., Partridge, S. R., van der Reijden, T., Dijkshoorn, L., and Iredell, J. (2007). Horizontal gene transfer in a polyclonal outbreak of carbapenem-resistant Acinetobacter baumannii. J. Clin. Microbiol. 45, 453-460. doi: 10.1128/JCM.01971-06

Wurtz, M. (1992). Bacteriophage structure. Electron Microsc. Rev. 5, 283-309. doi: 10.1016/0892-0354(92)90013-G

Xavier, B. B., Lammens, C., Ruhal, R., Kumar-Singh, S., Butaye, P., Goossens, H., et al. (2016). Identification of a novel plasmid-mediated colistin-resistance gene, $m c r-2$, in Escherichia coli, Belgium, June 2016. Euro Surveill. 21:30280. doi: 10.2807/1560-7917.ES.2016.21.27.30280

Yin, W., Li, H., Shen, Y., Liu, Z., Wang, S., Shen, Z., et al. (2017). Novel plasmid-mediated colistin resistance gene $\mathrm{mcr}-3$ in Escherichia coli. MBio 8:e00543-e00517. doi: 10.1128/mBio.00543-17

Zhang, C., Feng, Y., Liu, F., Jiang, H., Qu, Z., Lei, M., et al. (2017a). A phage-like IncY plasmid carrying the mor-1 gene in Escherichia coli from a pig farm in China. Antimicrob. Agents Chemother. 61:e02035-e02016. doi: 10.1128/AAC.02035-16

Zhang, R., Liu, L., Zhou, H., Chan, E. W., Li, J., Fang, Y., et al. (2017b). Nationwide surveillance of clinical carbapenem-resistant enterobacteriaceae (CRE) strains in China. EBioMed. 19, 98-106. doi: 10.1016/j.ebiom.2017.04.032

Zhang, Y., and LeJeune, J. T. (2008). Transduction of bla $a_{\mathrm{CMY}-2}$, tet(A), and tet(B) from Salmonella enterica subspecies enterica serovar Heidelberg to S. Typhimurium. Vet. Microbiol. 129, 418-425. doi: 10.1016/j.vetmic.2007.11.032

Zhao, F., and Zong, Z. (2016). Kluyvera ascorbata carrying the $m c r-1$ colistin resistance gene from hospital sewage. Antimicrob. Agents Chemother. 60, 7498-7501. doi: 10.1128/aac.01165-16

Zhao, F., Feng, Y., Lü, X., Mcnally, A., and Zong, Z. (2017). Remarkable diversity of Escherichia coli carrying mcr-1 from hospital sewage with the identification of two new $m c r-1$ variants. Front. Microbiol. 8:2094. doi: $10.3389 /$ fmicb.2017.02094

Conflict of Interest Statement: The authors declare that the research was conducted in the absence of any commercial or financial relationships that could be construed as a potential conflict of interest.

Copyright (๑) 2018 Zhou, Liu, Feng and Zong. This is an open-access article distributed under the terms of the Creative Commons Attribution License (CC BY). The use, distribution or reproduction in other forums is permitted, provided the original author(s) or licensor are credited and that the original publication in this journal is cited, in accordance with accepted academic practice. No use, distribution or reproduction is permitted which does not comply with these terms. 